03

\title{
Теплопроводность водонефтяных эмульсий обратного типа
}

\author{
(C) В.Г. Кучеров
}

Российский государственный университет нефти и газа (научно-исследовательский университет) им. И.М. Губкина, 119991 Москва, Россия

e-mail: vladimir@flotten.se

Поступило в Редакцию 15 марта 2020 г.

В окончательной редакции 20 марта 2020 г.

Принято к публикации 26 марта 2020 г.

Предложена корреляционная зависимость коэффициента теплопроводности бинарных дисперсных систем от их композиционного состава. Представлены результаты измерения коэффициента теплопроводности водонефтяных эмульсий обратного типа при давлении до $20 \mathrm{MPa}$ в диапазоне температур 293-353 К при изменении объемного содержания воды в эмульсии до $50 \%$. Полученная корреляционная зависимость пригодна для определения коэффициента теплопроводности эмульсий „вода-в-нефти“ в широком диапазоне изменения содержания воды и термобарических параметров.

Ключевые слова: нефти, водонефтяные эмульсии обратного типа, коэффициент теплопроводности, температура, давление.

DOI: $10.21883 / J T F .2020 .12 .50118 .14-20$

\section{Введение}

Для расчета коэффициента теплопроводности бинарных дисперсных систем в зависимости от их композиционного состава предложено значительное количество корреляционных зависимостей. Подробные обзор такого рода зависимостей приведен в [1-4]. Главными недостатками большинства этих зависимостей является наличие размерных коэффициентов, их применение зачастую ограничено определенным классом веществ или узким диапазоном термобарических параметров. Если результаты исследований теплопроводности твердых дисперсных материалов при изменении температуры и давления представлены в литературе [5,6], то теплопроводность эмульсий исследовалась только при атмосферном давлении [7-9].

\section{1. Корреляционная зависимость для расчета коэффициента теплопроводности бинарных дисперсных систем}

Используя анализа размерностей, в работе [10] был предложен подход к конструированию параметрическо-

Таблица 1. Физико-химические свойства и состав нефтей

\begin{tabular}{|c|c|c|c|c|c|}
\hline \multirow{2}{*}{ Свойства } & \multicolumn{5}{|c|}{ Номер образца } \\
\hline & 1 & 2 & 3 & 4 & 5 \\
\hline Плотность при 293 K, kg/m & 831 & 861 & 873 & 915 & 931 \\
\hline Молекулярная масса & 225 & 265 & 251 & 312 & 348 \\
\hline Температура начала кипения, K & 308 & 343 & - & 369 & 400 \\
\hline Вязкость при $323 \mathrm{~K}, \mathrm{~mm}^{2} / \mathrm{s}$ & 10.5 & 9.8 & 5.9 & $116^{*}$ & $462^{*}$ \\
\hline $\begin{array}{l}\text { Содержание, \% wt. } \\
\text { силикагелевых смол } \\
\text { асфальтенов } \\
\text { парафинов } \\
\text { воды }\end{array}$ & $\begin{array}{c}6.3 \\
1.0 \\
8.1 \\
-\end{array}$ & $\begin{array}{c}12 \\
2.8 \\
4.5 \\
-\end{array}$ & $\begin{array}{c}10 \\
2.2 \\
4.3 \\
-\end{array}$ & $\begin{array}{l}- \\
9.0 \\
2.6 \\
-\end{array}$ & $\begin{array}{c}16.4 \\
5.6 \\
1.0 \\
-\end{array}$ \\
\hline $\begin{array}{l}\text { Фракционный состав, \% vol. } \\
\text { до } 100^{\circ} \mathrm{C} \\
\text { до } 200^{\circ} \mathrm{C} \\
\text { до } 300^{\circ} \mathrm{C}\end{array}$ & $\begin{array}{l}10 \\
18 \\
20\end{array}$ & $\begin{array}{c}6 \\
22 \\
16\end{array}$ & $\begin{array}{c}7 \\
40 \\
18\end{array}$ & $\begin{array}{l}- \\
8.5 \\
19\end{array}$ & $\begin{array}{c}- \\
3.5 \\
19\end{array}$ \\
\hline
\end{tabular}

Примечани е. ${ }^{*}$ вязкость при $T=303 \mathrm{~K} .1-$ нефть Сосновского месторождения (Белоруссия), $2-$ нефть Каратюбинского месторождения (Казахстан), 3 - нефть месторождения Жонажол (Казахстан), 4 - нефть Вишнево-Полянского месторождения (Татарстан), 5 - нефть месторождения Каражанбас (Казахстан). 
Таблица 2. Экспериментальные значения коэффициента теплопроводности исследованных образцов нефтей, $\mathrm{W} /(\mathrm{m} \cdot \mathrm{K})$

\begin{tabular}{|c|c|c|c|c|}
\hline \multirow{2}{*}{$P, \mathrm{MPa}$} & \multicolumn{4}{|c|}{ Температура, K } \\
\hline & 293 & 313 & 333 & 353 \\
\hline \multicolumn{5}{|c|}{ нефть Сосновского месторождения } \\
\hline 0.1 & 0.122 & 0.117 & 0.108 & 0.101 \\
\hline 10 & 0.131 & 0.12 & 0.113 & 0.104 \\
\hline 20 & 0.136 & 0.127 & 0.12 & 0.108 \\
\hline \multicolumn{5}{|c|}{ нефть Каратюбинского месторождения } \\
\hline 0.1 & 0.127 & 0.121 & 0.115 & 0.104 \\
\hline 10 & 0.136 & 0.13 & 0.113 & 0.11 \\
\hline 20 & 0.14 & 0.136 & 0.131 & 0.116 \\
\hline \multicolumn{5}{|c|}{ нефть месторождения Жонажол } \\
\hline 0.1 & 0.133 & 0.127 & 0.116 & 0.110 \\
\hline 10 & 0.138 & 0.137 & 0.129 & 0.118 \\
\hline 20 & 0.146 & 0.144 & 0.140 & - \\
\hline \multicolumn{5}{|c|}{ Вишнево-Полянская нефть } \\
\hline 0.1 & 0.128 & 0.123 & 0.12 & 0.108 \\
\hline 10 & 0.135 & 0.134 & 0.131 & 0.127 \\
\hline 20 & 0.151 & 0.146 & 0.141 & 0.133 \\
\hline \multicolumn{5}{|c|}{ нефть месторождения Каражанбас } \\
\hline 0.1 & 0.13 & 0.126 & 0.12 & 0.115 \\
\hline 10 & 0.136 & 0.135 & 0.132 & 0.129 \\
\hline 20 & 0.153 & 0.15 & 0.142 & 0.135 \\
\hline
\end{tabular}

го класса функций для корреляционных зависимостей коэффициента теплопроводности бинарных дисперсных систем от их композиционного состава. При этом были сформулированы основные свойства, которыми должна обладать искомая зависимость:

$$
\lambda_{\Sigma}=F\left(\lambda_{1}, \lambda_{2}, \varphi_{1}, \varphi_{2}\right),
$$

где $\lambda_{\Sigma}, \lambda_{1}$ и $\lambda_{2}$ - теплопроводность смеси и ее компонентов; $\varphi_{1}$ и $\varphi_{2}$ - долевые концентрации компонентов, которые определены в виде объемных, массовых или мольных долей.

Во-первых, функция $F$ должнв быть четной относительно одновременной взаимной перестановки ее переменных $\lambda_{1}, \lambda_{2}$ и $\varphi_{1}, \varphi_{2}$, т. е. теплопроводность смеси не должна зависеть от способа ее приготовления:

$$
\lambda_{\Sigma}=F\left(\lambda_{1}, \lambda_{2}, \varphi_{1}, \varphi_{2}\right)=\lambda_{\Sigma}=F\left(\lambda_{2}, \lambda_{1}, \varphi_{2}, \varphi_{1}\right) .
$$

Во-вторых, функция $F$ должна удовлетворять следующим предельным свойствам при стремлении $\varphi_{1}$ или $\varphi_{2}$ к нулю или единице:

$$
\lambda_{1}=F\left(\lambda_{1}, \lambda_{2}, 0,1\right) \quad \text { и } \quad \lambda_{2}=F\left(\lambda_{1}, \lambda_{2}, 0,1\right) .
$$

И, наконец, если $\lambda_{1}<\lambda_{2}$ и функция $F$ монотонно изменяются при изменении переменной $\varphi_{2}$, тогда при любом соотношении компонентов рост доли второго компонента должен приводить к увеличению теплопроводности смеси. Математически это утверждение можно записать в следующем виде:

$$
\frac{\partial}{\partial \varphi_{2}} F\left(\lambda_{1}, \lambda_{2}, 1-\varphi_{2}, \varphi_{1}\right)\left\{\begin{array}{lll}
>0 & \text { if } & \lambda_{1}<\lambda_{2}, \\
=0 & \text { if } & \lambda_{1}=\lambda_{2}, \\
<0 & \text { if } & \lambda_{1}>\lambda_{2}
\end{array}\right.
$$

В итоге, как описано в $[10]$, конечный вид искомой корреляционной зависимости выглядит следующим образом:

$$
\begin{gathered}
\frac{\lambda_{\Sigma}}{\lambda_{1}}=\left(\frac{\lambda_{2}}{\lambda_{1}}\right)^{\varphi_{2}+\sum_{i=1}^{n} \alpha_{i} I_{i}\left(\varphi_{2}\right)}, \\
I_{i}\left(\varphi_{2}\right)=\int_{0}^{\varphi_{2}} \psi_{i}(\varphi) d \varphi,
\end{gathered}
$$

где $\alpha_{i}$ - эмпирические безразмерные коэффициенты, являющиеся функциями отношения $\lambda_{1} / \lambda_{2} ; I_{1}=\varphi_{1} \cdot \varphi_{2}$; $I_{2}=\varphi_{1} \cdot \varphi_{2} \cdot\left(\varphi_{1}-\varphi_{2}\right)$.

Зависимость (5) была проверена для водонефтяных эмульсий обратного типа „вода-в-нефти“. Водонефтяные эмульсии обратного типа относятся к однородным дисперсным смесям. Т.е. теплопроводность эмульсии при любом фиксированном компонентном составе не зависит от способа ее получения.

\section{2. Описание исследованных образцов}

Для исследования были отобраны пять образцов нефтей, существенно различающиеся по групповому составу и по основным физико-химическим свойствам. Образцы нефтей были предварительно обезвожены и дегазированы. В табл. 1 представлены физико-химические свойства исследованных образцов нефтей и их состав.

Эмульсии „вода-в-нефти“ приготавливались следующим образом. В мерный стакан, заполненный нефтью, помещался пропеллер высокооборотной мешалки. После включения мешалки в перемешиваемую нефть с помощью медицинского шприца постепенно добавлялось нужное количество дистиллированной воды. Полученная эмульсия перемешивалась со скоростью $10^{4} / \mathrm{min}$ в течение $10 \mathrm{~min}$. Скорость и время перемешивания определялись исходя из следующих соображений. Средний диаметр глобул воды в промысловых эмульсиях обратного типа составляет около $10 \mu \mathrm{m}$ [11]. Как показали предварительные эксперименты по приготовлению эмульсий, именно такой средний диаметр глобул получался при вышеуказанных параметрах приготовления. 
Таблица 3. Значения коэффициента теплопроводности водонефтяных эмульсий, $\mathrm{W} /(\mathrm{m} \cdot \mathrm{K})$

\begin{tabular}{|c|c|c|c|c|c|}
\hline \multirow{2}{*}{$\varphi_{2}$} & \multirow{2}{*}{$P, \mathrm{MPa}$} & \multicolumn{4}{|c|}{ Температура, K } \\
\hline & & 293 & 313 & 333 & 353 \\
\hline \multirow{4}{*}{0.1} & \multicolumn{5}{|c|}{ Эмульсия „вода-в-Сосновской нефти“ } \\
\hline & 0.1 & $\frac{0.145}{0.143(-1.3)}$ & $\frac{0.138}{0.138(0)}$ & $\frac{0.132}{0.129(-2.1)}$ & $\frac{0.123}{0.122(-0.8)}$ \\
\hline & 10 & $\frac{0.154}{0.153(0.8)}$ & $\frac{0.151}{0.142(-5.4)}$ & $\frac{0.138}{0.135(-2.3)}$ & $\frac{0.128}{0.125(2)}$ \\
\hline & 20 & $\frac{0.158}{0.158(0)}$ & $\frac{\underline{0.15}}{0.149(-0.3)}$ & $0.1 \frac{0.147}{42(-3.1)}$ & $\frac{0.136}{0.13(-4.5)}$ \\
\hline \multirow{3}{*}{0.5} & 0.1 & $\frac{0.272}{0.271(-0.4)}$ & $0.2 \frac{0.27}{271(0.4)}$ & $\frac{0.268}{0.266(-0.8)}$ & $\frac{0.259}{0.26(0.4)}$ \\
\hline & 10 & $0.28 \underline{0.29}$ & $\frac{0.286}{0.277(-3.1)}$ & $\frac{0.281}{0.273(-2.7)}$ & $0.2 \frac{0.271}{265(-2.1)}$ \\
\hline & 20 & $0 . \underline{0.306}$ & $\frac{0.304}{0.287(-5.6)}$ & $\frac{0.293}{0.283(-3.3)}$ & $\frac{0.282}{0.272(-3.6)}$ \\
\hline \multirow{4}{*}{0.1} & \multicolumn{5}{|c|}{ Эмульсия „вода-в-Каратюбинской нефти“ } \\
\hline & 0.1 & $\frac{0.151}{0.148(-1.7)}$ & $\frac{0.138}{0.143(3.4)}$ & $\frac{0.133}{0.137(2.9)}$ & $\frac{0.124}{0.125(1)}$ \\
\hline & 10 & $0 . \frac{0.158}{0.158(0)}$ & $0 . \frac{0.151}{0.152(1)}$ & $\frac{0.143}{0.135(-5)}$ & $\frac{0.138}{0.132(-4.4)}$ \\
\hline & 20 & $\frac{0.171}{0.162(-4.4)}$ & $\frac{0.159}{0.159(0)}$ & $0.1 \underline{0.15}$ & $\frac{0.141}{0.139(-1.8)}$ \\
\hline \multirow{3}{*}{0.5} & 0.1 & $\frac{0.279}{0.277(-0.8)}$ & $\frac{0.271}{0.276(-1.9)}$ & $0 . \underline{0.265}$ & $0 . \underline{0.263}$ \\
\hline & 10 & $\frac{0.295}{0.288(-2.2)}$ & $\frac{0.292}{0.288(-1.2)}$ & $\frac{0.281}{0.273(-2.7)}$ & $0 . \frac{0.272}{0.273(0.3)}$ \\
\hline & 20 & $0.295(-5.3)$ & $0.297(-1.7)$ & $0.296 \frac{0.3}{6(-1.3)}$ & $\frac{0.292}{0.282(-3.5)}$ \\
\hline \multirow{4}{*}{0.1} & \multicolumn{5}{|c|}{ Эмульсия „вода-в-Жонажольской нефти“ } \\
\hline & 0.1 & $\frac{0.16}{0.155(3.2)}$ & $0.149(-4.6)$ & $0.137(-3.3)$ & $\frac{0.126}{0.132(-4.9)}$ \\
\hline & 10 & $0 . \frac{0.164}{160(2.3)}$ & $\frac{0.156}{0.160(-2.3)}$ & $0.152(-3.3)$ & $\frac{0.134}{0.141(-4.9)}$ \\
\hline & 20 & $\frac{0.176}{0.169(3.9)}$ & $\frac{0.166}{0.167(-0.8)}$ & $\frac{0.156}{0.163(-4.8)}$ & - \\
\hline .5 & 0.1 & $0 \frac{0.283}{0.283(0)}$ & $\frac{0.275}{0.282(-2.7)}$ & $\frac{0.273}{0.275(-0.7)}$ & $\frac{0.259}{0.272(-4.8)}$ \\
\hline 0 & 10 & $0.2 \underline{0.289}$ & $\frac{\underline{0.284}}{0.296(-4.3)}$ & $\frac{0.277}{0.292(-5.4)}$ & $\frac{0.268}{0.283(-5.5)}$ \\
\hline & 20 & $0.30 \frac{0.3}{1(-0.2)}$ & $0.3 \underline{0.298}$ & $\frac{0.293}{0.306(-4.3)}$ & - \\
\hline
\end{tabular}


Таблица 3. (Продолжение).

\begin{tabular}{|c|c|c|c|c|c|}
\hline \multirow{2}{*}{$\varphi_{2}$} & \multirow{2}{*}{$P, \mathrm{MPa}$} & \multicolumn{4}{|c|}{ Температура, K } \\
\hline & & 293 & 313 & 333 & 353 \\
\hline \multirow{4}{*}{0.1} & \multicolumn{5}{|c|}{ Эмульсия „вода-в-Вишнево-Полянской нефти“ } \\
\hline & 0.1 & $\frac{0.147}{0.149(1.7)}$ & $\frac{0.146}{0.145(0.8)}$ & $\frac{0.138}{0.142(3)}$ & $\frac{0.13}{0.13(0)}$ \\
\hline & 10 & $\frac{0.158}{0.157(0.6)}$ & $\frac{0.15}{0.157(3.1)}$ & $\frac{0.149}{0.154(3.4)}$ & $\frac{0.14}{0.15(4.3)}$ \\
\hline & 20 & $\frac{0.167}{0.174(4.1)}$ & $\frac{0.16}{0.169(5.9)}$ & $\frac{0.156}{0.165(5.6)}$ & $\frac{0.148}{0.157(5.9)}$ \\
\hline \multirow{3}{*}{0.5} & 0.1 & $0 . \frac{0.273}{278(1.8)}$ & $\frac{0.268}{0.278(3.9)}$ & $0 \frac{0.259}{0.28(4.1)}$ & $\frac{0.255}{0.269(5.4)}$ \\
\hline & 10 & $0.2 \frac{0.293}{07(-1.9)}$ & $\frac{0.275}{0.293(5.7)}$ & - & - \\
\hline & 20 & $0 . \underline{0.309}$ & $\frac{0.304}{0.308(1.2)}$ & $\frac{0.292}{0.307(5.2)}$ & $\frac{0.285}{0.302(5.8)}$ \\
\hline \multirow{4}{*}{0.1} & \multicolumn{5}{|c|}{ Эмульсия „вода-в-Каражанбасской нефти“ } \\
\hline & 0.1 & $\frac{0.16}{0.152(5.3)}$ & $\frac{0.15}{0.148(1.3)}$ & $\frac{0.141}{0.142(-0.8)}$ & $\frac{0.138}{0.137(0.6)}$ \\
\hline & 10 & $\frac{0.163}{0.158(3,0)}$ & $\frac{0.158}{0.158(0)}$ & $\frac{0.15}{0.155(-3.4)}$ & $\frac{0.149}{0.152(-2.2)}$ \\
\hline & 20 & $\frac{0.173}{0.176(-1.7)}$ & $\frac{0.165}{0.174(-5.2)}$ & $0.1 \frac{0.157}{66(-5.6)}$ & $\frac{0.156}{0.159(-1.8)}$ \\
\hline \multirow{3}{*}{0.5} & 0.1 & $\frac{0.285}{0.28(1.8)}$ & 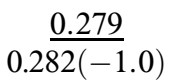 & $\frac{0.276}{0.28(-1.4)}$ & $0.2 \frac{0.263}{0.27(-5.5)}$ \\
\hline & 10 & $\frac{0.306}{0.288(5.7)}$ & 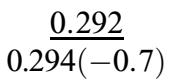 & $0 . \underline{0.283}$ & $\frac{0.281}{0.296(-5.2)}$ \\
\hline & 20 & $\frac{0.316}{0.308(2.5)}$ & $0.3 \underline{0.311}$ & $0 . \frac{0.306}{0.308(-0.7)}$ & $\frac{0.302}{0.304(-0 \ldots 6)}$ \\
\hline
\end{tabular}

Дальнейшее увеличение времени или скорости перемешивания не приводило к изменению среднего диаметра глобул. Полученная эмульсия подвергалась „старению“ (отстаивалась при комнатной температуре в течение 20 часов). Для того чтобы убедиться, что полученная эмульсия является эмульсией обратного типа, пробы ее до и после окончания „старения“ рассматривались под микроскопом. После проведения эксперимента по определению коэффициента теплопроводности пробы эмульсий из измерительных ячеек вновь рассматривались под микроскопом на предмет расслоения.

\section{3. Проведение экспериментов}

Измерение коэффициента теплопроводности проводилось с использованием калориметра, работающего по методу коаксиальных цилиндров. Подробное описание конструкции прибора, метода измерения, расчета погрешности измерений и методики проведения экспери- ментов изложены в [2]. Как показано в [2], максимальная относительная погрешность определения коэффициента теплопроводности для описанного выше метода не превышает 2.5\%. Проверочные эксперименты, проведенные с эталонной жидкостью (толуол), подтвердили, что отличия полученных на калориметре значений коэффициента теплопроводности от рекомендованных литературных значений лежат в пределах указанной погрешности.

В экспериментах сначала исследовались обезвоженные нефти, а затем водонефтяные эмульсии. Измерения коэффициента теплопроводности проводились на трех изобарах (0.1, 10 и $20 \mathrm{MPa})$ при температурах 293, 313, 333 и $353 \mathrm{~K}$. Содержание воды в эмульсиях составляло 10 и $50 \%$ vol.

\section{4. Обсуждение результатов}

Полученные данные по коэффициенту теплопроводности нефтей представлены в табл. 2. Эксперименталь- 
ные значения коэффициента теплопроводности эмульсий были сопоставлены с расчетными значениями, полученными по зависимости (5). Расчеты показали, что для описания теплопроводности исследованных эмульсий достаточно только одного члена степени в зависимости (5), т.е.

$$
\sum_{i=1}^{n} \alpha_{i} I_{i}\left(\varphi_{2}\right)=0 \quad \text { при } \quad n=0 .
$$

В этом случае получаем частный случай для зависимости (5). Полученное выражение совпадает с формулой Лихтенеккера [12] и выглядит следующим образом:

$$
\frac{\lambda_{\Sigma}}{\lambda_{1}}=\left(\frac{\lambda_{2}}{\lambda_{1}}\right)^{\varphi_{2}} .
$$

Результаты представлены в табл. 3. В числителе приведены значения коэффициента теплопроводности, полученные экспериментально, в знаменателе - рассчитанные по зависимости (6). В скобках даны отклонения расчетных значений от экспериментальных. Для эмульсий обратного типа $\varphi_{2}$ - объемная доля воды в эмульсии.

Как видно из табл. 2 и 3 коэффициент теплопроводности исследованных эмульсий при изменении содержания воды в эмульсии от 0 до 50\% увеличивается в $2.1-2.5$ раза.

При росте давления $\lambda$ эмульсий линейно увеличивается. Причем интенсивность роста значений коэффициента теплопроводности в среднем одинакова для эмульсий с различным содержанием воды и составляет $12 \%$ при росте давления от 0.1 до $20 \mathrm{MPa}$.

При повышении температуры коэффициент теплопроводности эмульсий уменьшается по линейному закону. При этом чем выше содержание воды в эмульсии, тем меньшее влияние оказывает температура на изменение $\lambda$. При водонасыщености $10 \%$ коэффициент теплопроводности эмульсий уменьшается в среднем на 15\% при изменении температуры от 293 до $353 \mathrm{~K}$, при содержании воды 50\% уменьшение составляет 7\% в том же температурном диапазоне. Это объясняется разнонаправленностью изменения коэффициентов теплопроводности нефти и воды при повышении температуры. Коэффициент теплопроводности нефти уменьшается с ростом температуры, а коэффициент теплопроводности воды растет.

Сопоставление значений коффициента теплопроводности, полученных экспериментально для эмульсий во всем термобарическом диапазоне и расчитанных по корреляционной зависимости (6), показывает их хорошую сходимость. Максимальная погрешность составляет $5.9 \%$, средняя $-2.6 \%$.

\section{Заключение}

В результате проведенного исследования получены значения коффициента теплопроводности водонефтяных эмульсий обратного типа в температурном интервале $293-353 \mathrm{~K}$ при изменении давления до $20 \mathrm{MPa}$. Показано, что полученная ранее автором корреляционная зависимость коэффициента теплопроводности однородных бинарных дисперсных систем от их композиционного состава пригодна для определения коэффициента теплопроводности эмульсий „вода-в-нефти“ в широком диапазоне изменения содержания воды и термобарических параметров.

\section{Конфликт интересов}

Автор заявляет, что у него нет конфликта интересов.

\section{Список литературы}

[1] Г.Н. Дульнев, Ю.П. Заричняк, В.В. Новиков. ИФЖ, XXXI, 150 (1976).

[2] Кучеров В.Г. Дисс. канд. техн. наук. М: МИНХ и ГП, 1987. $141 \mathrm{c}$.

[3] V.P. Privalko, V.V. Novikov. Adv. Polymer Sci., 119, 31 (1995).

[4] K. Pietrak, T.S. Wisniewski. J. Power Technol. 95 (1), 14 (2015).

[5] V.G. Kutcherov, B. Håkansson, R.G. Ross, G. Bäckström. J. Appl. Phys. 71, 1732 (1992).

[6] V. Kutcherov, A. Chernoutsan. J. Appl. Phys. 73, 2259 (1993).

[7] R.H. Wang, J.G. Knudsen. Ind. Eng. Chem. 50 (11), 1667 (1958).

[8] M. Yadegari, M. Seifi, J. Sabbaghzadeh. J. Thermormophysics and Heat Transfer. 26 (3), 536 (2012).

[9] T. Kawanami, K. Togashi, K. Fumoto, S. Hirano, P. Zhang, K. Shirai, S. Hirasawa. Energy. 117(P2), 562 (2016).

[10] В.И. Логинов, В.Г. Кучеров. ПМТФ. 4, 120 (1991).

[11] Г.А. Бабалян. Физико-химические процессы в добыче небти. (Недра, М., 1994) 200 с.

[12] K. Lichtenecker. Phys. Z. 10 (25), 1005 (1909). 